\title{
Anti-HER2 Bispecific Antibody-drug Conjugate ZW49
}

National Cancer Institute

\section{Source}

National Cancer Institute. Anti-HER2 Bispecific Antibody-drug Conjugate ZW49. NCI

Thesaurus. Code C162115.

An antibody-drug conjug ate (ADC) consisting of a bispecific monoclonal antibody (ZW25)

directed against two different epitopes of the tumor-associated antigen (TAA) human epidermal growth factor receptor 2 (HER2, receptor tyrosine-protein kinase erbB-2) linked to an as of yet undisclosed cytotoxic payload, with potential antineoplastic activity. Upon intravenous administration, anti-HER2 bispecific ADC ZW49 targets and binds to HER2 expressed on tumor cells. Following receptor internalization, the cytotoxic payload is released and induces tumor cell death through an as of yet unknown mechanism of action. Additionally, binding of HER2 may inhibit HER2 activation, HER2 signaling and HER2-mediated tumor cell growth. HER2, a tyrosine kinase receptor, is overexpressed by many cancer cell types. 